\title{
The Standard Model and Beyond
}

\author{
P. Fayet* \\ Laboratoire de Physique Théorique de l'Ecole Normale Supérieur \\ 24 rue Lhomond, 75231 Paris Cedex 05, France, \\ E-mail: 'fayet@physique.ens.
}

Abstract: We start with a brief presentation of the Standard Model and the weak neutral current, considering more specifically parity-violation effects in electron-hadron electroweak interactions. We then discuss some limitations of this model, motivating the consideration of larger frameworks such as grand-unification and supersymmetry. We also comment about the possible effects of additional light neutral gauge bosons.

\section{The Standard Model and the Weak Neutral Current}

What is the Standard Model of Particles and Interactions? And, at first, what are the particles and interactions we are talking about? Does this model give a satisfactory description of the real world? What did we learn from the discovery and study of Parity Violation in electronhadron interactions? Can we be satisfied with this Standard Model, or do we have reasons to go beyond it? If so, what sort of "new physics" could we expect, and how could it be tested? Here are some of the questions that we would like to address - although rather briefly - in this general introductory talk.

It is well known that the Standard Model provides a very good description of particle interactions. Here we are talking about weak, electromagnetic and strong interactions of known particles, essentially leptons and hadrons, the latter being strongly-interacting particles built from quarks and antiquarks. There are three charged leptons, the electron, the muon and the tau lepton, which all have their associated neutrinos, $\nu_{e}, \nu_{\mu}$ and $\nu_{\tau}$. We also know six sorts of quarks, after the top quark was finally discovered at Fermilab in 1994. They have electrical charges $\frac{2}{3}$ and $-\frac{1}{3}$, and may be associated two by two,

\footnotetext{
*Unité Propre du CNRS, associée à l'Ecole Normale Supérieure et à l'Université de Paris-Sud
}

as indicated in Table. We leave momentar-

\begin{tabular}{|l}
6 leptons: $\quad\left(\begin{array}{c}\nu_{e} \\
e^{-}\end{array}\right)\left(\begin{array}{c}\nu_{\mu} \\
\mu^{-}\end{array}\right)\left(\begin{array}{l}\nu_{\tau} \\
\tau^{-}\end{array}\right)$ \\
\hline 6 quarks: $\quad\left(\begin{array}{l}u \\
d\end{array}\right) \quad\left(\begin{array}{l}c \\
s\end{array}\right)\left(\begin{array}{l}t \\
b\end{array}\right)$ \\
\hline
\end{tabular}

Table 1: The three families of leptons and quarks. Left-handed lepton and quark fields transform as members of $S U(2)$ electroweak doublets, while their right-handed counterparts are singlets.

ily aside the fourth interaction, gravitation, very well described - although only at the classical level - by Einstein's theory of General Relativity. At the level of individual particles it is extremely weak compared to the three other interactions, at all energies in which we shall be interested, as indicated by the extremely small value of the Newton constant, $G_{\text {Newton }} \simeq 10^{-38} \mathrm{GeV}^{-2}$.

Weak, electromagnetic and strong interactions are now well understood from the exchanges of spin-1 mediators known as gauge bosons, between the "constituents of matter", as are often called the spin- $\frac{1}{2}$ leptons and quarks. The spin1 mediators are the famous gauge bosons of the Standard Model: the photon, whose exchanges between charged particles are responsible for the 
electromagnetic interactions. The eight gluons of Quantum Chromodynamics (QCD), whose exchanges between "colored" quarks (each quark appearing as a triplet of a color $S U(3)$ gauge group) are responsible for the strong interactions, binding together quarks three by three to form protons $(u u d)$ and neutrons $(d d u)$. And the intermediate gauge bosons $W^{ \pm}$and $Z$, very heavy - approximately 80 and $91 \mathrm{GeV} / c^{2}$, respectively - responsible for the various "chargedcurrent" and "neutral-current" weak-interaction processes $^{1}$.

These $W^{ \pm}$and $Z$ appear, together with the photon, as the four gauge bosons of the $S U(2) \times$ $U(1)$ gauge symmetry group of the electroweak

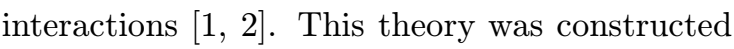
in the sixties and early seventies, by extending to weak and strong interactions the ideas of gauge invariance underlying the very successful theory of quantum electrodynamics (QED). This one is the prototype of a renormalisable theory, in which quantum effects can be computed in a consistent way and are found to be finite, order by order in perturbation theory.

If we go back to the sixties, a severe problem was posed by the definition of a quantum theory for the weak interactions. While these interactions were well described, at the classical level, by the Fermi theory involving local products of four fermion fields (times a coupling constant proportional to the Fermi constant, $G_{F} \simeq$ $10^{-5} \mathrm{GeV}^{-2}$ ), it was not possible to compute

\footnotetext{
${ }^{1}$ More precisely, currently used values of the $W^{ \pm}$and $Z$ masses are $m_{W}=80.4 \pm .1 \mathrm{GeV} / c^{2}$, and $m_{Z}=$ $91.187 \pm .003 \mathrm{GeV} / c^{2}$. The fine-structure constant of electromagnetic interactions is $\alpha=e^{2} / 4 \pi \epsilon_{0} \hbar c \simeq$ $1 / 137.036$; its value at the $Z$ mass is given by $\alpha^{-1}\left(m_{Z}\right)=128.9 \pm .1$. The Fermi coupling constant of the weak interactions is $G_{F}=$ (1.16639 \pm $.00001) 10^{-5} \mathrm{GeV}^{-2}(\hbar c)^{3}$; and the electroweak mixing angle, to be introduced later (defined by $\tan \theta=g^{\prime} / g$ with $g$ and $g^{\prime}$ evaluated at the $Z$ mass) is given by $\sin ^{2} \theta \simeq .2314 \pm .0003$

The QCD strong interaction coupling $\alpha_{s}=g_{s}^{2} / 4 \pi$ "runs" with the energy, decreasing from large values at low energies, down to $\alpha_{s}\left(m_{Z}\right) \simeq .12$. The Newton constant of gravitational interactions is $G_{N} \simeq$ $(.6707 \pm .0001) \quad 10^{-38}\left(\mathrm{GeV} / c^{2}\right)^{-2}(\hbar c)$, which corresponds to a "Planck mass", $m_{P}=\sqrt{\hbar c / G_{N}} \simeq$ $1.22110^{19} \mathrm{GeV} / c^{2} \simeq 2.17710^{-5} \mathrm{~g}$, and a "Planck length" $L_{P} \simeq \hbar /\left(m_{P} c\right) \simeq 1.61610^{-33} \mathrm{~cm}$.
}

higher-order quantum corrections to weakinteraction processes, found to be severely divergent. The solution to this problem went through the understanding of (charged-current) weak interactions as due to the exchanges of charged heavy intermediate bosons named $W^{+}$and $W^{-}$, coupled, proportionally to some dimensionless constant $g$, to a linear combination of charged vector and axial-vector currents. The Fermi constant $G_{F}$ may then be expressed in terms of this coupling $g$ and the mass of the intermediate bosons $W^{ \pm}$, according to the formula

$$
\frac{G_{F}}{\sqrt{2}}=\frac{g^{2}}{8 m_{W}^{2}} .
$$

But this was not enough to provide a sensible quantum theory. It appeared also necessary to view these spin-1 intermediate bosons $W^{+}$and $W^{-}$

as gauge bosons coupled to charged currents, with a spontaneous symmetry breaking mechanism at work to provide these gauge bosons a mass (otherwise they would remain exactly massless, just like the photon, as a consequence of the con-

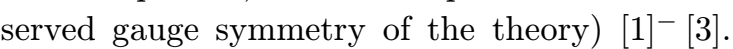
The corresponding gauge group had to be related with the $U(1)$ gauge group of quantum electrodynamics, since the $W^{ \pm}$'s are charged and must interact with the photon. Using simply $S U(2)$ (with $U(1)_{\mathrm{QED}}$ as a subgroup) would not have provided a really satisfactory solution; nowadays we can simply see, for example, that it would not allow for quarks having fractional electrical charges $\frac{2}{3}$ and $-\frac{1}{3}$. This led to the consideration by Glashow, Salam, Ward and Weinberg of $S U(2) \times U(1)$ as the proposed (spontaneously broken) gauge symmetry group of electromagnetic and weak interactions. These interactions become, in some sense, partly unified within the framework of a single "electroweak" theory.

But $S U(2) \times U(1)$ has four generators, the "weak-isospin" generators $T_{1}, T_{2}$ and $T_{3}$, and the "weak hypercharge" $Y$, not only three. This required, if the idea was to be right, the existence of an additional neutral gauge boson, the $Z-$ which had to be very heavy - coupled to a new "weak neutral current". At the same time the massless photon field $A$ is coupled to the elec- 
tromagnetic current, associated with the electric charge now expressed, in terms of the elementary unit $e$, by

$$
Q=T_{3}+\frac{Y}{2}
$$

More precisely, if we denote by $W_{1}, W_{2}, W_{3}$ and $B$ the four gauge fields of $S U(2) \times U(1)$, the charged $W$ fields are given by

$$
W^{ \pm}=\frac{W_{1} \mp i W_{2}}{\sqrt{2}},
$$

while the fields of the new weak neutral boson $Z$ and of the photon $\gamma$ are given by the two orthogonal linear combinations

$$
\left\{\begin{array}{l}
Z=\cos \theta W_{3}-\sin \theta B \\
A=\sin \theta W_{3}+\cos \theta B .
\end{array}\right.
$$

$\theta$ is the electroweak mixing angle, fixed in terms of the two $S U(2)$ and $U(1)$ gauge coupling constants $g$ and $g^{\prime}$ by

$$
\tan \theta=\frac{g^{\prime}}{g},
$$

the elementary charge being given by

$$
e=g \sin \theta=g^{\prime} \cos \theta \text {. }
$$

The new weak neutral current coupled to the $Z$ boson is then expressed $^{2}$,

in terms of the weak isospin and electromagnetic currents, by

$$
J_{Z}=J_{3}-\sin ^{2} \theta J_{\mathrm{em}} .
$$

${ }^{2}$ The couplings of the neutral gauge bosons to chiral quark and lepton fields $\psi_{L, R}=\frac{1 \mp \gamma_{5}}{2} \psi$ are obtained from the Lagrangian density

$\mathcal{L}=\overline{\psi_{L, R}}\left[i \partial_{\mu}-\left(g T_{3} W_{\mu 3}+\frac{g^{\prime}}{2} Y B_{\mu}\right)\right] \gamma^{\mu} \psi_{L, R}$, by rediagonalizing the couplings of the neutral gauge fields $W_{\mu 3}$ and $B_{\mu}$ to the corresponding weak isospin and weak hypercharge currents, as follows:

$$
\begin{aligned}
& g J_{3}^{\mu} W_{\mu 3}+\frac{g^{\prime}}{2} J_{Y}^{\mu} B_{\mu}= \\
& \sqrt{g^{2}+g^{\prime 2}}\left(J_{Z}^{\mu}=J_{3}^{\mu}-\sin ^{2} \theta J_{\mathrm{em}}^{\mu}\right) Z_{\mu} \\
& \quad+g \sin \theta\left(J_{\mathrm{em}}^{\mu}=J_{3}^{\mu}+\frac{1}{2} J_{Y}^{\mu}\right) A_{\mu} .
\end{aligned}
$$

This leads to expressions $(\overline{1} \cdot \overline{1} \cdot \overline{2})$ and $\left(\overline{1}^{-} \cdot \overline{6_{1}^{\prime}}\right)$ of the charge operator $Q=T_{3}+\frac{Y}{2}$ and the elementary charge $e=g \sin \theta$, and to expression (‥1. $\left.\vec{Z}_{1}\right)$ of the weak neutral current $J_{Z}$.
This shows the crucial rôle played, in the framework of the standard model and its neutral current phenomenology, by the electroweak mixing angle $\theta$ and the associated $\sin ^{2} \theta$.

The new weak neutral current $J_{Z}$ should then manifest itself through a new type of neutrino-scattering processes, such as $\stackrel{(-)}{\nu}_{\mu}+e^{-} \rightarrow$ $\stackrel{(-)}{\nu}_{\mu}+e^{-}$, or $\stackrel{(-)}{\nu}_{\mu}+$ nucleon $\rightarrow \stackrel{(-)}{\nu}_{\mu}+X$, which were effectively discovered [4]-4] at CERN and Fermilab, in 1973-1974. The $W^{ \pm}$and $Z$ bosons themselves could be directly produced in $p \bar{p}$ collisions [i $[\overline{1}-1]$ (at CERN in 1983, and later at Fermilab) and $e^{+} e^{-}$annihilations (at LEP and SLC), providing a further confirmation of the ideas of gauge theories through the direct observation of the massive gauge bosons.

Remember, however, that one does not only have to determine the value of $\sin ^{2} \theta$ in the standard model, despite the great importance of this parameter. It was necessary, in the first place, to establish the validity of this model. Now that this is done (to a very large extent) and that the corresponding value of $\sin ^{2} \theta(\simeq .231)$ is precisely known, it is essential to pursue the analysis of the radiative corrections, in connection with the understanding of the electroweak symmetry breaking mechanism and the searches for spin-0 Higgs bosons. And, at the same time, to look for possible deviations which might signal the existence of "new physics" beyond the standard model. For all these purposes it is crucial to have different types of experiments, performed at different energies, to study the various aspects of neutral-current interactions, not only in neutrino scatterings, but also in electron interactions with matter.

\section{Neutral currents in electron- hadron interactions}

\subsection{Neutral-current effects and parity vio- lation in electron-hadron interactions}

Neutral current effects should be present in the interactions of electrons with matter, but are then in competition with ordinary electromagnetic interactions, which are normally much larger, as 
long as the center-of-mass energies and momenta remain small compared to the $W^{ \pm}$or $Z$ masses. Indeed $\gamma$-exchange electromagnetic amplitudes are proportional to $e^{2} / q^{2}$, while $Z$-exchange weakneutral-current amplitudes are proportional to $G_{F} \simeq 10^{-5} \mathrm{GeV}^{-2}$, with the $W^{ \pm}$and $Z$ masses - which verify, at the classical level, $m_{Z}=$ $m_{W} / \cos \theta-$ related to $G_{F}$ by

$$
\frac{G_{F}}{\sqrt{2}}=\frac{g^{2}}{8 m_{W}^{2}}=\frac{g^{2}+g^{\prime 2}}{8 m_{Z}^{2}} .
$$

Weak-interaction amplitudes, very small at low energies owing to the very small value of the Fermi constant $G_{F}$, become comparable in magnitude with electromagnetic amplitudes, for large values of the center-of-mass energies and momenta of the order of the $W^{ \pm}$and $Z$ masses. At low or even moderately-high energies, however, the large effects of electromagnetic interactions could well prevent one to detect the effects of the weak-neutral-current interactions between electrons and hadrons. For example neutral-current amplitudes, proportional to $G_{F}$, are very roughly of the order of $10^{-4}$ times electromagnetic amplitudes, for values of the momentum transfer $\left|q^{2}\right| \approx 1(\mathrm{GeV} / c)^{2}$.

But weak interactions violate the discrete symmetry of parity, which exchanges the left and right orientations in space. This is reflected by the fact that the charged weak currents to which the $W^{ \pm}$'s are coupled are not pure vector currents as the electromagnetic current, but are given by a $V-A$ combination of vector and axial parts. This also corresponds to the fact that left-handed quark and lepton fields are associated in $S U(2)$ doublets, as indicated in Table, while their right-handed counterparts are $S U(2)$ singlets. The $S U(2) \times U(1)$ structure of the electroweak theory requires that the new weak neutral current coupled to the $Z$ boson, given by $J_{Z}=J_{3}-\sin ^{2} \theta J_{\mathrm{em}}$, must also violate parity.

It is then possible to take advantage of the fact that the interference terms between electromagnetic and weak-neutral-current amplitudes in electron-hadron interactions violate parity, to be able to detect the effects of the latter at moderately high energies; and even also, in a more surprising way, at very low energies! Actually parity-violation effects in electron-hadron interactions had already been considered by Zel'dovich [i]'], as early as in 1958, before the construction of the standard model. Such interference effects between weak and electromagnetic amplitudes were indeed observed in a famous SLAC experiment [i7] in 1978, in which a small asymmetry $\left(\approx 10^{-4}\right)$ in the deep-inelastic scattering of polarized electrons on deuterium could be measured.

Furthermore, neutral-current interactions can also induce mixings between atomic levels of different parities, an effect that is enhanced in heavy atoms and may then become accessible to observations, as discussed by M.A. and C. Bouchiat [iיi] in their well-known paper of 1974. These parityviolation effects in atomic physics, still very tiny, could indeed be detected and are now precisely measured by several experiments performed with different heavy atoms, cesium, bismuth, lead and thallium.

Both electron-hadron-scattering and atomic physics experiments give complementary informations on the four coefficients $c_{1 u}$ and $c_{2 u}$, $c_{1 d}$ and $c_{2 d}$, generally used to parametrize the parity-violating part of the effective Lagrangian density describing the neutral-current interactions of the electrons with the $u$ and $d$ quarks, main constituents of the protons and neutrons. The parity-violating part of this low-energy effective Lagrangian density may be written ${ }^{3}$ as follows:

$$
\begin{aligned}
& \mathcal{L}_{\mathrm{eff}}=\frac{G_{F}}{\sqrt{2}} \Sigma_{q=u, d}\left[\begin{array}{cccc}
c_{1 q} & \bar{q} \gamma^{\mu} q & \bar{e} \gamma_{\mu} \gamma_{5} e
\end{array}\right. \\
& \left.+c_{2 q} \bar{q} \gamma^{\mu} \gamma_{5} q \quad \bar{e} \gamma_{\mu} e\right] \text {. }
\end{aligned}
$$

\subsection{Parity violation in electron-hadron scat- tering experiments}

The weak-neutral-current amplitudes corresponding to this effective Lagrangian density interfere

\footnotetext{
${ }^{3}$ See also, however, subsection ${ }_{1}^{1}$, if relatively light neutral gauge bosons are present, in addition to the heavy $Z$ boson. Then the $c_{i q}^{\prime} s$ don't have to be the same for different experiments performed at different values of $q^{2}$ ! Light $U$ bosons with parity-violating interactions were initially discussed in supersymmetric theories with an extra $U(1)$ gauge group, but their possible existence should be considered independently of this original motivation.
} 
with ordinary electromagnetic amplitudes, induced by the exchanges of a photon between the $u$ or $d$ quarks (of charges $\frac{2}{3}$ and $-\frac{1}{3}$ ) and the electron. For the SLAC experiment [i] [i] with an isoscalar deuterium target,

$$
e_{R, L}^{-}+\text {deuterium } \rightarrow e^{-}+X,
$$

performed with polarized electrons of energies $\approx$ $20 \mathrm{GeV}$, and momentum transfer $Q^{2}=-q^{2}$ (defined to be positive) of the order of $\approx 1.6(\mathrm{GeV} / c)^{2}$, the interference term in the deep-inelastic scattering cross-section on $u$ and $d$ quarks is a linear combination of the two quantities $\left(\frac{2}{3}\right) c_{i u}+\left(-\frac{1}{3}\right) c_{i d}$, i.e.

$$
c_{1 u}-\frac{1}{2} c_{1 d} \quad \text { and } \quad c_{2 u}-\frac{1}{2} c_{2 d} .
$$

We can easily compute an approximate value of the asymmetry by neglecting the contribution of the $c_{2 q}$ 's (which are small in the standard model, for $\left.\sin ^{2} \theta \simeq \frac{1}{4}\right)^{4}$. More precisely, the asymmetry $^{5} \quad A=\frac{\sigma_{R}-\sigma_{L}}{\sigma_{R}+\sigma_{L}}$, proportional to $\frac{G_{F}}{\alpha / q^{2}}$, is given by:

$$
\begin{aligned}
\frac{A}{Q^{2}} & =\frac{3 G_{F}}{5 \sqrt{2} \pi \alpha}\left[\left(c_{1 u}-\frac{1}{2} c_{1 d}\right)\right. \\
& \left.+\left(c_{2 u}-\frac{1}{2} c_{2 d}\right) \frac{1-(1-y)^{2}}{1+(1-y)^{2}}\right]
\end{aligned}
$$

in which $y(\sim .2)$ is the fraction of the incoming electron energy transferred to the hadrons in the

\footnotetext{
${ }^{4}$ In this approximation both electromagnetic and weak amplitudes for the scattering of electrons on $u$ and $d$ quarks involve the quark vector current $\bar{q} \gamma^{\mu} q$. The polarized cross-sections may be written as:

$$
\begin{aligned}
\sigma_{R, L} & \propto\left[\frac{2}{3} \frac{-e^{2}}{q^{2}} \pm \frac{G_{F}}{\sqrt{2}} c_{1 u}\right]^{2} \\
& +\left[-\frac{1}{3} \frac{-e^{2}}{q^{2}} \pm \frac{G_{F}}{\sqrt{2}} c_{1 d}\right]^{2} .
\end{aligned}
$$

The interference terms between electromagnetic and weak amplitudes generate an asymmetry

$$
\begin{aligned}
A & =\frac{\sigma_{R}-\sigma_{L}}{\sigma_{R}+\sigma_{L}}=2 \frac{G_{F}}{\sqrt{2}} \frac{Q^{2}}{e^{2}} \frac{\frac{2}{3} c_{1 u}-\frac{1}{3} c_{1 d}}{\left(\frac{2}{3}\right)^{2}+\left(-\frac{1}{3}\right)^{2}} \\
& =\frac{3 G_{F} Q^{2}}{5 \sqrt{2} \pi \alpha}\left(c_{1 u}-\frac{1}{2} c_{1 d}\right) .
\end{aligned}
$$

With $\frac{3 G_{F}}{5 \sqrt{2} \pi \alpha} \simeq 2.1610^{-4}(\mathrm{GeV})^{-2}$, and $\left(c_{1 u}-\right.$ $\left.\frac{1}{2} c_{1 d}\right) \simeq-.36$ in the standard model, we get an approximate value of $A / Q^{2} \simeq-810^{-5}(\mathrm{GeV} / c)^{-2}$.
}

${ }^{5}$ The polarized cross-sections on $u$ and $d$ quarks may final state. The measured value of the asymmetry, given by

$$
A / Q^{2}=(-9.5 \pm 1.6) 10^{-5}(\mathrm{GeV} / c)^{-2}
$$

, has been used to discrimate the standard model from a number of possible alternatives. With a further analysis of the $y$ dependence of the asymmetry $(2.5)$, these experiments provided combined constraints on the two quantities $\left(c_{1 u}-\frac{1}{2} c_{1 d}\right)$ and $\left(c_{2 u}-\frac{1}{2} c_{2 d}\right)$. And also, in the framework of the standard model (for which the $c_{i q}$ 's are known functions of $\sin ^{2} \theta$, given in subsection 2. $\overline{4}$ ), a determination of $\sin ^{2} \theta \simeq .224 \pm .020$.

We refer, more generally, to ref. 9 for a description of the different electron-hadron scattering experiments that have been performed.

\subsection{Parity-violation in atomic physics ex- periments}

In atomic physics, for which a typical momentum transfer may be taken as $q \sim m_{e} c \alpha$, one might naïvely anticipate parity-violation effects to be of the order of $\frac{G_{F}}{\alpha / q^{2}}$, leading to rough expectations $\sim G_{F} m_{e}^{2} \alpha \sim 10^{-14}$, considerably smaller than the $\sim 10^{-4}$ at $\left|q^{2}\right| \sim 1(\mathrm{GeV} / c)^{2}$ that we just discussed for the SLAC experiment.

For a heavy atom however [8], the momentum transfer corresponding to a penetrating electron in the vicinity of a nucleus of charge $Z$ is

$$
\begin{aligned}
\text { be written as: } & \\
\sigma_{R, L}^{u} & \propto\left[\frac{2}{3} \frac{-e^{2}}{q^{2}} \pm \frac{G_{F}}{\sqrt{2}}\left(c_{1 u}+c_{2 u}\right)\right]^{2} \\
& +\left[\frac{2}{3} \frac{-e^{2}}{q^{2}} \pm \frac{G_{F}}{\sqrt{2}}\left(c_{1 u}-c_{2 u}\right)\right]^{2}(1-y)^{2} \\
\sigma_{R, L}^{d} & \propto\left[-\frac{1}{3} \frac{-e^{2}}{q^{2}} \pm \frac{G_{F}}{\sqrt{2}}\left(c_{1 d}+c_{2 d}\right)\right]^{2} \\
& +\left[-\frac{1}{3} \frac{-e^{2}}{q^{2}} \pm \frac{G_{F}}{\sqrt{2}}\left(c_{1 d}-c_{2 d}\right)\right]^{2}(1-y)^{2} .
\end{aligned}
$$

The interference terms between electromagnetic and weak amplitudes generate an asymmetry

$$
\begin{aligned}
A & =2 \frac{G_{F}}{\sqrt{2}} \frac{Q^{2}}{e^{2}} \frac{\frac{2}{3}\left[\left(c_{1 u}+c_{2 u}\right)+\left(c_{1 u}-c_{2 u}\right)(1-y)^{2}\right]}{\left[\left(\frac{2}{3}\right)^{2}+\left(-\frac{1}{3}\right)^{2}\right]\left[1+(1-y)^{2}\right]} \\
& -\frac{\frac{1}{3}\left[\left(c_{1 d}+c_{2 d}\right)+\left(c_{1 d}-c_{2 d}\right)(1-y)^{2}\right]}{\left[\left(\frac{2}{3}\right)^{2}+\left(-\frac{1}{3}\right)^{2}\right]\left[1+(1-y)^{2}\right]} .
\end{aligned}
$$

This leads directly to expression $\left(\underline{2} \cdot \overline{L_{1}^{\prime}}\right)$. 
$q \sim m_{e} c Z \alpha$, and this electron interacts coherently with the constituents of the nucleus. As a result the previous estimate, now replaced by $\frac{Z G_{F}}{\alpha / q^{2}} \sim G_{F} m_{e}^{2} \alpha Z^{3}$, is enhanced by a factor which behaves roughly like $Z^{3}$.

More precisely, parity-violation effects in atomic physics are mostly sensitive to the vector part of the quark weak-neutral-current, combined with the axial part of the electronic current, which can induce mixings between atomic levels of different parities. This term involving the vector part of the quark weak-neutral-current in the effective Lagrangian density (2.2) is parametrized by the two coefficients $c_{1 u}$ and $c_{1 d}$, while a nucleus with $Z$ protons and $N$ neutrons includes $(2 Z+N) u$ quarks and $(Z+2 N) d$ quarks.

In the non-relativistic limit for which the electron may be described by a two-component spinor $\varphi$ (with small components expressed as $\chi \simeq$ $\left.\frac{\vec{\sigma} \cdot \vec{p}}{2 m_{e}} \varphi\right)$, the relevant part in the parity-violating Lagrangian density $(\overline{2} .2 i)$ reads:

$$
\begin{aligned}
\mathcal{L}_{\mathrm{P} . \mathrm{V} .}^{(1)} & \simeq \frac{G_{F}}{\sqrt{2}}\left[(2 Z+N) c_{1 u}+(Z+2 N) c_{1 d}\right] \\
& \cdot \delta^{3}(\vec{r}) \quad\left(\varphi^{\dagger} \frac{\vec{\sigma} \cdot \vec{p}}{2 m_{e}} \varphi+\text { c.c. }\right)
\end{aligned}
$$

In terms of the "weak charge" of the nucleus, defined as

$Q_{W}=-2\left[c_{1 u}(2 Z+N)+c_{1 d}(Z+2 N)\right]$

this corresponds to the following effective parityviolating potential between an electron and a nucleus:

$$
V_{\mathrm{P} . \mathrm{V} .}^{(1)} \simeq \frac{G_{F}}{4 \sqrt{2}} \frac{Q_{W}}{m_{e}}\left(\vec{\sigma} \cdot \vec{p} \delta^{3}(\vec{r})+\delta^{3}(\vec{r}) \vec{\sigma} \cdot \vec{p}\right) .
$$

(The other part in the effective Lagrangian density $(2.2 i)$, parametrized by the $c_{2 q}$ coefficients, involves the axial quark currents and leads to additional parity-violating contributions, which are proportional to the spin of the nucleus.)

Due to the very small value of the Fermi constant (corresponding to $G_{F} m_{e}^{2} \alpha \simeq 210^{-14}$ ) the parity-violation effects might have been totally negligible at low energies. But as shown by the Bouchiat's they are significantly enhanced in heavy atoms, since the matrix elements of $V_{\mathrm{P} . \mathrm{V}}^{(1)}$. between two $S$ and $P$ states mixed by this parityviolating interaction involve the product of a $s$ wave function near the origin (which behaves like $\sqrt{Z}$ ), times the derivative of a $p$ wave function (which behaves like $Z \sqrt{Z}$ ), times the weak charge $Q_{W}$ (which behaves like $Z$ ).

Owing to the resulting $\approx Z^{3}$ enhancement factor, in particular, these parity-violation effects become accessible to experiments, and have now been measured, for several heavy atoms, at a level of precision that can be better that $1 \%$ [in]. The results are well in agreement with the values expected from standard model calculations. In the case of cesium $(Z=55$ with $Z+N=133)$ for example, the measured and "standard model" values of the weak charge [1인, $\left.\underline{1}_{1}^{1} \underline{1}_{1}^{\prime}\right]$ are given below, and verify:

$$
\begin{aligned}
Q_{W \exp } & -Q_{W \mathrm{sM}}=\left(-72.4 \pm 0.3_{\exp } \pm 0.8_{\mathrm{th}}\right) \\
& -(-73.1 \pm 0.1) \simeq 0.7 \pm 0.9 .
\end{aligned}
$$

For heavy atoms expression (2.7) of $Q_{W}$ corresponds to a linear combination of $c_{1 u}$ and $c_{1 d}$ that is quite different (and very roughly orthogonal) to the linear combination $\left(c_{1 u}-\frac{1}{2} c_{1 d}\right)$ to which the SLAC experiment is sensitive. The two types of experiments then play complementary rôles in the determination of the exact structure of the electron-hadron weak-neutral-current interactions.

\subsection{Parity-violation and the neutral cur- rent in the Standard Model}

In the standard model, there is a single weak neutral current, given by $J_{Z}=J_{3}-\sin ^{2} \theta J_{\mathrm{em}}$. The corresponding contributions of the electron, and the $u$ and $d$ quarks, to this weak neutral current, read as follows:

$$
\left(J_{Z}^{\mu}\right)_{e}=\left(-\frac{1}{4}+\sin ^{2} \theta\right) \bar{e} \gamma^{\mu} e+\frac{1}{4} \bar{e} \gamma^{\mu} \gamma_{5} e,
$$

for the electronic weak neutral current, and

$$
\left\{\begin{array}{rrrr}
\left(J_{Z}^{\mu}\right)_{u} & = & \left(+\frac{1}{4}-\frac{2}{3} \sin ^{2} \theta\right) \bar{u} \gamma^{\mu} u \\
& - & \frac{1}{4} \bar{u} \gamma^{\mu} \gamma_{5} u, \\
\left(J_{Z}^{\mu}\right)_{d} & = & \left(-\frac{1}{4}+\frac{1}{3} \sin ^{2} \theta\right) \bar{d} \gamma^{\mu} d \\
& + & \frac{1}{4} \bar{d} \gamma^{\mu} \gamma_{5} d,
\end{array}\right.
$$


for the $u$ and $d$ quark weak neutral currents.

From the effective Lagrangian density

$$
\begin{aligned}
\mathcal{L}_{\text {eff }} & =-\frac{g^{2}+g^{\prime 2}}{m_{Z}^{2}}\left(J_{Z}^{\mu}\right)_{q}\left(J_{\mu Z}\right)_{e} \\
& =-8 \frac{G_{F}}{\sqrt{2}}\left(J_{Z}^{\mu}\right)_{q}\left(J_{\mu} Z\right)_{e},
\end{aligned}
$$

we get the expressions (at the classical level) of the four coefficients $c_{i u}$ and $c_{i d}$ which parametrize the effective Lagrangian density $\left(\left[\begin{array}{l}2.21 \\ -2\end{array}\right)\right.$ :

$$
\left\{\begin{aligned}
c_{1 u} & \simeq-\frac{1}{2}+\frac{4}{3} \sin ^{2} \theta, \\
c_{1 d} & \simeq \frac{1}{2}-\frac{2}{3} \sin ^{2} \theta, \\
c_{2 u} & \simeq-\frac{1}{2}+2 \sin ^{2} \theta, \\
c_{2 d} & \simeq \frac{1}{2}-2 \sin ^{2} \theta .
\end{aligned}\right.
$$

And the weak charge $Q_{W}$ defined by eq. (12.7) is given (again up to small higher-order corrections) by the following expression ${ }^{6}$, which depends on $\sin ^{2} \theta$ :

$$
Q_{W} \simeq Z\left(1-4 \sin ^{2} \theta\right)-N .
$$

To fix ideas, a rough evaluation of the $c_{i q}$ parameters would be, for $\sin ^{2} \theta \simeq .225, c_{1 u} \simeq$ $-.20, c_{1 d} \simeq .35, c_{2 u} \simeq-.05, c_{2 d} \simeq .05$. And the weak charge would then be given by $Q_{W} \simeq .1 Z-N \simeq-72.5$, in the case of cesium (for which $Z=55$ and $N=78$ ), which practically coincides with the measured value given in eq. $(2.9 i)$. But precise estimates require the consideration of radiative corrections.

Electron-hadron scattering and atomic physics experiments led, in the framework of the Standard Model, to precise determinations of the $\sin ^{2} \theta$ parameter [9.9]. Maybe even more importantly, they played a crucial rôle in discriminating this model from possible alternatives, through the determination of the exact structure of the

\footnotetext{
${ }^{6}$ This may also be obtained directly in the standard model, from the effective Lagrangian density

$$
\mathcal{L}_{\mathrm{P} . \mathrm{V} .}^{(1)}=-2 \frac{G_{F}}{\sqrt{2}}\left(J_{Z}^{\mu}\right)_{q \text { vect. }} \bar{e} \gamma_{\mu} \gamma_{5} e .
$$

The vector part in the hadronic $Z$ current leads to a density $\left[\frac{1}{4}(Z-N)-\sin ^{2} \theta \underline{Z}\right] \delta^{3}(\vec{r})$, and therefore to the parity-violating potential (2.8), with expression (2.14) of the weak charge $Q_{W}$.
}

weak neutral current. More precisely one had to establish whether all experiments involving weak-neutral-current effects, including of course neutrino and $e^{+} e^{-}$scattering experiments, could be interpreted, or not, in terms of a single weak neutral current; and if so, if this neutral current did actually obey eq. (1.7.), $J_{Z}=J_{3}-$ $\sin ^{2} \theta J_{\mathrm{em}}$, and for which value of $\sin ^{2} \theta$.

Up to now only one weak neutral current $J_{Z}$, obeying eq. (1.7), was exhibited experimentally. And the experimental determinations of the weak-neutral-current interaction parameters [1]1] are in good agreement with the standard model values (here evaluated for $m_{\mathrm{Higgs}} \simeq m_{Z}$ ). One has also, in addition, $c_{2 u}+c_{2 d} \simeq-.0095$, in the standard model.

On the other hand many theoretical ideas involve additional weak neutral currents coupled to extra neutral gauge bosons. The possible existence of such additional gauge bosons is frequently suggested, for example in left-right symmetric or grand-unified theories, and in theories with extra $U(1)$ gauge groups, often motivated by supersymmetry or superstring ideas. These ideas of left-right symmetry (with gauge group $\left.S U(3) \times S U(2)_{L} \times S U(2)_{R} \times U(1)_{B-L}\right)$ and grand-unification (with larger gauge groups such as $O(10)$ or $E(6))$ also provide natural frameworks in which neutrinos could have tiny masses and oscillate from one flavor to another.

It is worth to emphasize, at this stage, that experiments working at low values of the momentum transfer - and in particular atomic physics experiments - could be sensitive to neutral gauge bosons $(U)$ that would be both relatively light and very weakly coupled, thereby escaping detection in experiments performed higher $\left|q^{2}\right|$ large compared to $m_{U}^{2}$. We shall return to this point in subsection i 6.

\section{Electroweak physics and precision tests of the Standard Model}

Altogether many experimental data have confirmed the validity of the Standard Model (al- 


\begin{tabular}{|c|c|c|}
\hline & experiment & standard model \\
\hline$c_{1 u}$ & $-.216 \pm .046$ & $-.1885 \pm .0003$ \\
$c_{1 d}$ & $.361 \pm .041$ & $.3412 \pm .0002$ \\
$c_{2 u}-\frac{1}{2} c_{2 d}$ & $-.03 \pm .12$ & $-.0488 \pm .0008$ \\
\hline$Q_{W}(\mathrm{Cs})$ & $-72.41 \pm .25 \pm .80$ & $-73.12 \pm .06$ \\
$Q_{W}(\mathrm{Tl})$ & $-114.8 \pm 1.2 \pm 3.4$ & $-116.7 \pm .1$ \\
\hline
\end{tabular}

Table 2:

though we still have no detailed xperimental information about the mechanism by which the $S U(2) \times U(1)$ electroweak symmetry gets broken). Let us mention, in particular, the direct production of the $W^{ \pm}$and $Z$ bosons at the CERN and Fermilab $p \bar{p}$ colliders, the detailed study of the properties of the $Z$ boson at LEP 1 and SLC, and, more recently, the direct production of $W^{ \pm}$pairs at LEP 2. Also the top quark, necessary for the consistency of the theory, was found at Fermilab with a mass $m_{\text {top }} \simeq$ $170-180 \mathrm{GeV} / c^{2}$, in agreement with the expectations derived from the analysis of the radiative corrections.

The standard model of strong, electromagnetic and weak interactions gives, at the present time, a remarkably good description of the interactions of particles, very well understood from the exchanges of spin-1 gauge bosons between spin- $1 / 2$ leptons and quarks ${ }^{7}$. The consistency of all experimental results with this Standard Model, for a value of the electroweak mixing angle given by $\sin ^{2} \theta \simeq .231$, now requires that one takes into account the effects of electroweak radiative corrections. And experiments are getting sensitive to the effects of spin-0 Higgs bosons associated with the spontaneous breaking of the electroweak symmetry, a point to which we shall

\footnotetext{
${ }^{7}$ We leave aside the question of neutrino masses and oscillations, two phenomena which are absent from the standard model defined stricto sensu, but may be incorporated easily through the introduction of right-handed neutrino fields.
}

return later. The running of the strong interaction coupling constant $\alpha_{s}$, measured up to LEP energies $\left(\alpha_{s}\left(m_{Z}\right) \simeq .12\right)$, is also in good agreement with QCD expectations.

The precise determination of these numbers, including the electromagnetic constant $\alpha\left(m_{Z}\right) \simeq$ $1 / 129$, is essential in the discussion of a possible unification of the three (suitably-normalized) $S U(3), S U(2)$ and $U(1)$ gauge couplings at high energies, as expected in grand-unified theories. As we shall see in section $15, \overline{5}$, such an unification does not occur if the evolution of the couplings from "low" energies (i.e. $\approx m_{Z}$ ) to very-high energies is computed with the particle spectrum of the standard model. On the other hand a unification of the values of the three gauge couplings is obtained, at an energy of the order of $10^{16}$ $\mathrm{GeV} / c^{2}$ or so, if the evolution of the three gauge couplings is governed by the particle spectrum of the Supersymmetric Standard Model. This is often taken as a possible (indirect) indication in favor of supersymmetry. Before discussing briefly such extensions of the Standard Model, let us say a few things about Higgs bosons.

In the Standard Model the spontaneous breaking of the electroweak gauge symmetry is induced by fundamental spin-0 fields known as (EnglertBrout) Higgs fields [1] istence of a new neutral spin 0 particle called the Higgs boson. This particular aspect of the Standard Model has not been confirmed experimentally yet, and one can still question the actual 
existence of such a particle, which remains the only missing ingredient of the model, after the experimental discovery of the top quark.

Since the Higgs boson has escaped all direct searches performed at LEP up to now, its mass must be larger than about $80 \mathrm{GeV} / c^{2}$, a limit that recently increased up to about $90 \mathrm{GeV} / c^{2}$ and even more, with the increase in the energies accessible at LEP 2. Can the mass of this particle, essential for the renormalisability of the theory, be very large? Then we could no longer think of the Higgs boson as an ordinary elementary particle: one has $\Gamma_{\text {Higgs }} \approx \frac{1}{2} m_{H}^{3}$, if the Higgs mass and width are measured in TeV's, so that a $1 \mathrm{TeV} / c^{2}$ Higgs would have a width of about $\frac{1}{2} \mathrm{TeV} / c^{2}$ !

Moreover the coupling constant $\lambda$ which governs the magnitude of the Higgs boson selfinteractions grows like the square of the Higgs mass $^{8}$, so that, if we want to be able to compute perturbatively up to energies of the order of the Higgs mass at least, this mass should be less than about $800 \mathrm{GeV} / c^{2}$ or so. (If, furthermore, we demand to be able to compute perturbatively up to a large grand-unification scale of the order of $10^{16} \mathrm{GeV} / c^{2}$, the Higgs mass should be less than about $200 \mathrm{GeV} / c^{2}$; it should also, at the same time, be larger than about $120 \mathrm{GeV} / c^{2}$, if the standard model vacuum state considered is to remain stable or at least metastable.)

${ }^{8}$ More precisely, if we write the potential of the spin- 0 doublet Higgs field $\varphi$ as

$$
V(\varphi)=\lambda\left(\varphi^{\dagger} \varphi\right)^{2}-\mu^{2} \varphi^{\dagger} \varphi,
$$

the neutral component of the Higgs field acquires a nonvanishing vacuum expectation value $\left\langle\varphi^{0}\right\rangle=\frac{v}{\sqrt{2}}$, with $v=\sqrt{\frac{\mu^{2}}{\lambda}}$. The $W^{ \pm}$and $Z$ masses are $m_{W}=$ $g v / 2, m_{Z}=\sqrt{g^{2}+g^{\prime 2}} v / 2$, so that

$$
\frac{G_{F}}{\sqrt{2}}=\frac{g^{2}}{8 m_{W}^{2}}=\frac{g^{2}+g^{\prime 2}}{8 m_{Z}^{2}}=\frac{1}{2 v^{2}},
$$

which determines $v=\left(G_{F} \sqrt{2}\right)^{-\frac{1}{2}} \simeq 246 \mathrm{GeV}$. The Higgs mass being given by $m_{H}^{2}=2 \mu^{2}$, one can express the Higgs self-coupling constant as

$$
\lambda=\frac{\mu^{2}}{v^{2}}=\frac{G_{F}}{\sqrt{2}} m_{H}^{2} .
$$

In particular $\lambda^{2} / 4 \pi \lesssim 1$, for $m_{H} \lesssim \pi^{\frac{1}{4}} 2 v \simeq 650$ $\mathrm{GeV}$.
However, unlike in the case of the top quark, lowest-order radiative corrections to the presently measured quantities are not very sensitive to the Higgs boson mass (their dependence in $m_{H}$ being generally logarithmic, rather than quadratic as in the case of $\left.m_{\mathrm{top}}\right)$. At the present stage, the analysis of radiative corrections indicates that the Higgs mass should be less than about 300 $\mathrm{GeV} / c^{2}$, in the framework of the standard model.

Should one expect a future discovery of this elusive particle, and then consider it as the final confirmation of an achieved and satisfactory theoretical construction? Unfortunately not, since, despite the remarkable achievements of this model, it cannot be considered as a complete and satisfactory theory of the fundamental laws of Nature. Indeed the standard model leaves, on the theoretical side, many questions unanswered.

\section{Open questions in the Standard Model}

\subsection{The origin of the charges, and the ques- tion of parity conservation}

Why is the electric charge quantized, and the quark charges $+2 / 3$ and $-1 / 3$ (times the elementary unit charge $e)$ ? These values, given by $Q=T_{3}+\frac{Y}{2}$, follow from appropriate choices for the weak hypercharges $Y$ of the chiral quark and lepton fields: $Y=-1$ for the left-handed electron field $e^{-}$, but -2 for $e_{R}^{-} ; Y=1 / 3$ for the quark fields $u_{L}$ and $d_{L}$, but $4 / 3$ and $-2 / 3$ for $u_{R}$ and $d_{R}$. Why these rather special values? And at first, why is parity violated in weak interactions (left-handed quark and lepton fields being members of $S U(2)$ doublets, and righthanded ones singlets), while it remains conserved by electromagnetic ones? This is well described by the standard model, but we would like to understand the origin of the $S U(3) \times S U(2) \times U(1)$ gauge symmetry properties of quark and lepton fields.

\subsection{The "family problem" and the origin of the lepton and quark mass spectrum}

Why are there three families of leptons and quarks $\left(\nu_{e}, e, u, d\right),\left(\nu_{\mu}, \mu, c, s\right),\left(\nu_{\tau}, \tau, t, b\right)$, rather 
than a single one, and how could their mass spectrum and mixing angles be theoretically understood? This is in fact an old question, which dates back to the discovery of the muon. Is there a symmetry between the three families, and if so, why and how gets it broken? Are neutrinos exactly massless - as in the standard model defined stricto sensu - or slightly massive? In that case, do they "oscillate" from one flavor to another, $\nu_{e}$ 's, for example, being transformed into $\nu_{\mu}$ 's or $\nu_{\tau}$ 's , and conversely? Could such oscillations be responsible for the deficit of the measured flux of neutrinos produced by the Sun, compared to expectations based on the standard model of the Sun? Could $\nu_{\mu} \rightarrow \nu_{\tau}$ oscillations, for example, be responsible for the anomalies presented by the observed fluxes of atmospheric neutrinos [i1 2i]? Could massive neutrinos, or other particles, provide some of the "dark matter" that seems to be present in the Universe?

\subsection{The $C P$ problem, and the origin of matter in the Universe}

The $C P$ symmetry which relates matter to antimatter - while exchanging the left and right orientations in space - is a symmetry for almost but not all interactions, since it is slightly violated in weak decays of neutral kaons. This $C P$ violation is generally attributed to the effect of the (weak) $C P$-violating phase parameter $\delta$ in the Cabibbo-Kobayashi-Maskawa mixing matrix between the three quark families, although this remains to be proven. Once $C P$ is broken it has no reason to be an exact symmetry of the strong interactions, and the neutron should be expected to have some electric dipole moment. Since none has been found the corresponding amount of "strong" $C P$-violation, measured by the effective dimensionless parameter $\theta_{\mathrm{QCD}}$, should be smaller than $\approx 10^{-9}$. Why should $\theta_{\mathrm{QCD}}$ be so small? A possible explanation involves extensions of the standard model with a new broken $U(1)$ symmetry called the PecceiQuinn symmetry. This would require the existence of a neutral, very light, spin-0 Goldstonelike particle, the axion [1] 3 , decaying into photon pairs. But none has been observed, and its existence is rather constrained, both from parti- cle physics experiments and astrophysical arguments.

A related question concerns the density of matter in the Universe. Why is the average number of nucleons of the order of a few $10^{-10}$ compared to the number of photons in the primordial $2.7^{\circ} \mathrm{K}$ cosmic microwave background radiation? What is the origin of these nucleons? Are they remnants of the annihilation of matter with antimatter in the very early Universe, an extremely small excess of matter being present since the origin of time? If not, could this very small excess of matter have been generated from an initially symmetric Universe? As noted by Sakharov [1 [14"] in 1966, this would require the existence of interactions that do not conserve the baryon number $B$, so as to generate a net excess of nucleons; and do not conserve the charge conjugation $C$ and $C P$ symmetries, so that matter and antimatter can evolve asymmetrically, through out of equilibrium phenomena. What could be these $B, C$ and $C P$-violating interactions? Perhaps those of a "grand-unified" theory relating quarks to leptons; or maybe those which should appear at very high energies within the standard model itself, when non-perturbative effects of electroweak interactions are taken into account?

\section{4 "Too many arbitrary parameters"}

The standard model depends in fact, not on a single parameter $\sin ^{2} \theta$, but on a total of twenty arbitrary parameters: the three gauge coupling constants $g_{s}, g$ and $g^{\prime}$ of the $S U(3) \times S U(2) \times$ $U(1)$ gauge group; the two parameters $\mu^{2}$ and $\lambda$ which determine the mass and self-interactions of the Higgs boson; the nine quark and chargedlepton masses, plus the three quark-mixing angles and the (weak) $C P$-violating phase $\delta$; with also, in addition, the (strong) $C P$-violating parameter $\theta_{\mathrm{QCD}}$ and the analogous parameter $\theta_{2}$ of $S U(2)$. In a satisfactory theory these parameters should not appear as totally free, and there should be a way to understand the values taken.

This is at the starting point of the grandunification approach [1 15], in particular. Can one relate the values of the three gauge coupling constants of $S U(3), S U(2)$ and $U(1)$ ? and establish relations between quark and lepton masses? 
In grand-unified theories of strong, electromagnetic and weak interactions, $S U(3), S U(2)$ and $U(1)$ appear as three subgroups of a single gauge group - like $S U(5)$ or $O(10)$ - so that the three gauge couplings of the standard model can be related. This grand-unification of strong, electromagnetic and weak interactions should typically occur at very high energies initially thought to be of the order of $10^{14} \mathrm{GeV}$ and now, more likely, in the framework of supersymmetric theories, $10^{16} \mathrm{GeV}$. Quarks are related with leptons, and the proton is generally expected to be unstable, but with a very long lifetime. Independently of this idea of grand-unification, the Higgs bosons and their interaction potential may be constrained in the framework of supersymmetric theories, in which bosons and fermions are related, and where one is led to postulate the existence of new superpartners for all particles $[1 \overline{1} \overline{6}, \overline{1} \overline{1} \overline{7}]$. Supersymmetric and grand-unified theories will be discussed in section 5

\subsection{The problem of quantum gravity}

Gravitational interactions are well described, classically, by the theory of general relativity, for which the space-time is no longer flat but curved, the sources of the curvature being the densities and fluxes of energy and momentum. But gravity poses a severe problem when one tries to include it within the framework of quantum physics. Quantum gravity is not a renormalizable theory (nor a finite one), since the Newton constant $G_{N} \simeq$ $10^{-38} \mathrm{GeV}^{-2}$ has dimension -2 , in contrast with the gauge coupling constants, which are dimensionless. The higher orders we go in perturbation theory, the more and more divergent are the various successive amplitudes we would like to evaluate.

Of course for individual particles gravitational interactions are essentially negligible at all energies up to several TeV's and even much more, due to the extremely small value of the Newton constant. But since gravitational interactions act on energies and momenta, their effective intensity behaves roughly like $G_{N} E^{2}$, growing quadratically with the energy. They should then have a strong intensity $(\sim 1)$ at huge energies of the order of the Planck energy, $E_{P}=\sqrt{\frac{\hbar c^{5}}{G_{N}}} \simeq$
$10^{19} \mathrm{GeV}$. The troublesome ill-defined quantum gravity effects are expected to become important, and even essential, at such huge energies. It is, however, very tempting to ignore them completely at much lower energies. Still one has to deal with this very fundamental question, but finding a consistent quantum theory of gravity remains an extremely hard problem. One hopes to deal with this difficulty by abandoning the idea of pointlike particles, in favor of extended objects like strings [1] $\left.\overline{1}_{1}\right]$ or membranes. In any case this indicates that the standard model, even extended to include classical gravity, cannot be regarded as a satisfactory theory of all interactions.

Supersymmetric theories offer a natural way to introduce gravitation in particle physics, in the sense that a supersymmetric theory of particles, once the supersymmetry is realized locally, necessarily includes general relativity, and describes also the gravitational interactions of the particles considered. Furthermore supersymmetry seems to be a necessary ingredient for the consistency of string theories and other theories of extended objects, that might provide a solution to the problems of quantum gravity.

\subsection{Another problem of gravity: the value of the cosmological constant $\Lambda$}

In general relativity, the energy density of the vacuum has a definite meaning, since it couples to gravity; it can be defined and parametrized through a quantity called the cosmological constant, $\Lambda=8 \pi G_{N} \rho_{\mathrm{vac}}$. Cosmological constraints on $\Lambda$ are very severe, even more if they are expressed in terms of the natural length scale associated with general relativity and gravitation, $L_{\text {Planck }}=\hbar /\left(m_{\mathrm{P}} c\right) \simeq 1.610^{-33} \mathrm{~cm}$ :

$$
\begin{aligned}
|\Lambda| & <\left(610^{9} \text { light-years }\right)^{-2} \simeq 310^{-56} \mathrm{~cm}^{-2} \\
& \simeq 10^{-121} L_{\text {Planck }}^{-2} .
\end{aligned}
$$

This constraint, which corresponds to $\left|\Omega_{\Lambda}\right|=$ $\left|\rho_{\text {vac }} / \rho_{c}\right|<2, \quad$ implies that the corresponding "vacuum energy density" $\rho_{\text {vac }}$ should be smaller than about twice the critical density of the Universe $\left(\rho_{c}=3 H_{0}^{2} / 8 \pi G_{N} \simeq .510^{-5} \mathrm{GeV} / \mathrm{cm}^{3} \simeq\right.$ 
$10^{-29} \mathrm{~g} / \mathrm{cm}^{3}$, with $c=1$ ), i.e.

$\left|\rho_{\text {vac }}\right|<10^{-5} \mathrm{GeV} / \mathrm{cm}^{3} \simeq 10^{-46} \mathrm{GeV}^{4}$.

But before that, as the Universe expanded and cooled, it ought to have gone through various phase transitions. During the electroweak transition, $\rho_{\text {vac }}$ should have changed by some $10^{8} \mathrm{GeV}^{4}$, and again by some $10^{-4} \mathrm{GeV}^{4}$ during the QCD "deconfining" transition. Furthermore, the naïve expectation would normally have been $\sim 10^{76} \mathrm{GeV}^{4}$, with the vacuum quantum fluctuations of gravity cut-off at the Planck scale. Why is the current value of the vacuum energy density so close to zero, compared to any of the above natural scales? Some principle seems necessary to explain the very small value of the cosmological constant $\Lambda$. Here again supersymmetry may have a role to play, since bosons and fermions give opposite contributions to the vacuum energy density so that the cosmological constant naturally vanishes in a supersymmetric theory, although the problem generally reappears when the supersymmetry gets broken.

\subsection{The problem of the hierarchy of mass scales}

This "hierarchy problem" is not a problem of the standard model itself, but a difficulty encountered when one tries to embed it into a bigger theory involving large mass scales, such as the grand-unification or the Planck scales. These large scales tend to contaminate the electroweak scale associated with the $W^{ \pm}$and $Z$ bosons, which would make the latter very heavy. Indeed the parameter $\mu$ of the standard model which enters in the determination of the electroweak scale then tends to be naturally very large, since it usually appears as an algebraic sum of extremely large quantities. How can $\mu$ be so small with respect to much larger scales? This generally requires unnatural adjustments of parameters (first performed at the tree approximation, then redone again, order by order in perturbation theory), known as "fine-tuning". This is rather unsatisfactory, unless it may be cured by means of some principle (as in supersymmetry) allowing scalar fields to remain light.
We shall not discuss the attempts which have been made, as in technicolor and other models, to avoid the introduction of fundamental spin-0 Higgs fields responsible for the electroweak breaking (these fields being replaced by bilinear products of fermion fields acquiring non-vanishing vacuum expectation values). Technicolor models, in particular, face a number of difficulties, and do not seem in agreement with experimental data. Nor shall we discuss the possibility that quarks and leptons, and maybe even gauge bosons, might be composite, since it seems difficult to pursue very far in this direction. We shall now concentrate on the approaches of supersymmetry and grand unification, which seem the most promising ones to overcome the various limitations of the standard model, despite the fact that no direct experimental confirmation of these ideas has been found yet.

\section{About Supersymmetry and Grand Unification}

\subsection{Supersymmetry}

Supersymmetry is at first an algebraic structure [16], involving a spin- $\frac{1}{2}$ fermionic symmetry generator $Q$ satisfying the algebra:

$$
\left\{\begin{array}{ccc}
\{Q, \bar{Q}\} & =-2 \gamma_{\mu} P^{\mu} \\
{\left[Q, P^{\mu}\right]} & = & 0
\end{array}\right.
$$

This spin- $\frac{1}{2}$ supersymmetry generator $Q$ can potentially relate bosons and fermions in a physical theory, provided we succeed in identifying physical bosonic and fermionic fields that might be related under such a symmetry. The presence of the generator of spacetime translations $P^{\mu}$ in the right handside of the anticommutation relations is at the origin of the relation of supersymmetry with general relativity and gravitation, since a locally supersymmetric theory must necessarily be invariant under local coordinate transformations.

But which bosons and fermions could be related by such a supersymmetry? Perhaps the photon with the neutrino (more precisely, one 
of the three neutrinos $\nu_{e}, \nu_{\mu}$ and $\nu_{\tau}$, or conceivably a linear combination of them)? or the charged $W^{-}$with the electron? or maybe gluons with quarks ...? Alas, we quickly find out that the supersymmetry algebra is of no use to establish direct relations between known bosons and fermions, such as those we just mentioned. If we do not want to abandon this otherwise rather appealing idea, we have to make a further physical assumption, the superpartner hypothesis [1 $1 \overline{7}_{1}$. The photon cannot be related with any of the known neutrinos, but with a "neutrino" of a new type, in some sort a "neutrino of the photon", which I called the photino. In a similar way gluons have to be associated with a color-octet of (self-conjugate) spin- $\frac{1}{2}$ Majorana fermions called the gluinos - in spite of the old prejudice, now forgotten, according to which no such particles should exist. Leptons and quarks should be associated with new bosonic partners taken as spin0 (rather than spin-1) particles, called sleptons and squarks.

Altogether all ordinary particles (quarks, leptons, gauge bosons, and Higgs bosons if they do exist) should be associated with new ones, still unobserved, that we call their superpartners. Furthermore, in addition to this general doubling of the number of particle states, the spontaneous breaking of the electroweak symmetry should be induced in this framework, not by a single doublet of Higgs fields as in the Standard Model, but by a pair of them [17]. This implies the existence of a pair of charged Higgs bosons $\left(H^{ \pm}\right)$, and of several neutral ones. Altogether this leads to what is known as the "Supersymmetric Standard Model", describing the interactions of the $8+3+1$ gauge superfields of $S U(3) \times S U(2) \times U(1)$ with quark and lepton superfields, and two doublet Higgs superfields responsible for quark, lepton, and $W^{ \pm}$and $Z$ masses. Its minimal particle content is given in Table plicity further mixings between the various "neutralinos" described by mixtures of neutral gaugino and higgsino fields). Note that each quark $q$ or charged lepton $l$ of spin $\frac{1}{2}$ is associated with two spin-0 partners collectively denoted by $\tilde{q}$ or $\tilde{l}$, while a left-handed neutrino $\nu_{L}$ is associated with a single sneutrino $\tilde{\nu}$.
One of the problems one had to face, in the early days of supersymmetry, before it could be applied to the description of the real world, as indicated above, was the fact that baryon and lepton numbers, perfectly conserved in all known physical processes, are carried by fundamental fermions only (the familiar spin- $\frac{1}{2}$ quarks and leptons), and not by fundamental bosons. This feature cannot be maintained in a supersymmetric theory. To overcome the obstacle it appeared necessary to attribute baryon and lepton numbers to bosonic fields as well as to fermionic ones. These newly-introduced bosonic fields are precisely the squark and slepton fields $\tilde{q}$ and $\tilde{l}$, a denomination which makes obvious the fact that they must carry baryon and lepton numbers.

Still the consideration of such new bosonic fields carrying baryon or lepton numbers can be a source of additional difficulties. In particular known interactions are due to the exchanges of spin-1 intermediate gauge bosons (gluons, photons, $W^{ \pm}$'s and $Z$ 's), not of spin-0 particles. In the presence of many new spin-0 particles carrying baryon and lepton numbers one runs into the risk of generating additional unwanted interactions mediated by these new spin-0 bosons. This problem is naturally avoided, however, if the new squarks and sleptons have no direct Yukawa couplings to ordinary fermions (quarks and leptons) [i 1 ;]. This is indeed the case if the Lagrangian density involves only even functions of the various quark and lepton superfields, as that must be if we are to define conserved baryon and lepton numbers. This can be formulated in terms of a new symmetry principle, related with the definitions of baryon and lepton numbers.

We were thus led to distinguish between two large classes of particles, depending on the parity character of a new quantum number called $R$. Ordinary particles are $R$-even, and their superpartners $R$-odd - the supersymmetry generator relating the two classes of particles being itself an $R$-odd operator. The corresponding multiplicative quantum number, called $R$-parity, is +1 for ordinary particles, -1 for their superpartners. This original definition can be reexpressed easily in terms of the spin $S$ of a particle, and 


\begin{tabular}{|c|c|c|}
\hline Spin 1 & Spin $1 / 2$ & Spin 0 \\
\hline $\begin{array}{l}\text { gluons } g \\
\text { photon } \gamma\end{array}$ & $\begin{aligned} \text { gluinos } & \tilde{g} \\
\text { photino } & \tilde{\gamma}\end{aligned}$ & \\
\hline $\begin{array}{c}W^{ \pm} \\
Z\end{array}$ & $\begin{aligned} \operatorname{winos} & \widetilde{W}_{i}^{ \pm} \\
\operatorname{zinos} & \widetilde{Z}_{i} \\
\text { higgsino } & \tilde{h}^{0}\end{aligned}$ & $\left.\begin{array}{c}H^{ \pm} \\
H \\
h, A\end{array}\right\} \begin{array}{l}\text { Higgs } \\
\text { bosons }\end{array}$ \\
\hline & $\begin{array}{ll}\text { leptons } & l \\
\text { quarks } & q\end{array}$ & $\begin{array}{ll}\text { sleptons } & \tilde{l} \\
\text { squarks } & \tilde{q}\end{array}$ \\
\hline
\end{tabular}

Table 3: Minimal particle content of the Supersymmetric Standard Model.

of baryon and lepton numbers, as follows:

$$
R \text {-parity }=(-1)^{2 S}(-1)^{3 B+L} .
$$

$R$-parity, if it actually is a symmetry of the Lagragian density, forbids direct couplings of the new spin-0 squarks and sleptons to ordinary spin$\frac{1}{2}$ quarks and leptons. Expression (5.2i) of $R$ parity illustrates that violations of the $R$-parity symmetry would necessarily imply violations of baryon and/or lepton number conservation laws, with the risk of generating unwanted processes such as a much too fast proton decay mediated by $R$-odd squark exchanges, if both $B$ and $L$ violations are simultaneously allowed ${ }^{9}$.

Whether or not it turns out to be absolutely conserved, $R$-parity is essential in the discussion of the physics of supersymmetric theories. Superpartners can only be pair-produced if $R$-parity

\footnotetext{
${ }^{9}$ Of course in grand-unified theories, in which quarks are related to leptons, $B$ and $L$ no longer have to be separately conserved, and the proton is normally expected to decay, into $\pi^{0} e^{+}$for example. But $B$ and $L$-violating processes are then mediated by new particles having very large masses of the order of the grand-unification scale, so that the resulting proton lifetime is very long. This would not be the case if $B$ and $L$ violations were induced by $R$-odd particles with much smaller masses, so that such violations cannot be tolerated. Let us note, in addition, that the equivalent expression

$$
R \text {-parity }=(-1)^{2 S}(-1)^{3(B-L)}
$$

shows that $R$-parity may still be conserved even if $B$ and $L$ are separately violated, as long as their difference $B-L$ remains conserved, even only modulo 2 .
}

is conserved. (Even if $R$-parity were not conserved by some of the interaction terms in the Lagrangian density, superpartners would still be expected to be pair-produced in most cases.) Furthermore, most superpartners are in general expected to be unstable, with ( $R$-parity-conserving) decay modes such as

$$
\begin{gathered}
\tilde{l} \rightarrow l+\text { neutralino }, \\
\tilde{q} \rightarrow q+\text { gluino (or neutralino) } \\
\text { gluino } \rightarrow q \bar{q}+\text { neutralino , etc. }
\end{gathered}
$$

for example.

An absolute conservation of $R$-parity also implies that the lightest superpartner, called the LSP, should be absolutely stable. This one is presumably neutral, the best candidate being a photino or more generally is a mixture of the various neutral spin- $\frac{1}{2}$ fermions called neutralinos. (A stable positively charged LSP, on the other hand, would lead to superheavy isotopes of hydrogen, which have not been observed [1] $\overline{1}$ |.) This LSP is a very good candidate to constitute, at least for a part, the non-baryonic Dark Matter that seems to be present in our Universe. Missing energy carried away by two unobserved LSP's is one of the most characteristic features of the pair production of "supersymmetric particles", if $R$-parity is conserved.

One can still consider the possibility of $R$ parity-violating theories in which $B$ would be conserved but not $L$ (or conversely), although 
this would imply a somewhat dissymmetric treatment of quarks and leptons. The proton would then still remain stable, despite the presence of $R$-parity-violating interactions. Let us also mention that the squarks of a supersymmetric theory with $R$-parity violations have been advocated as a possible explanation for the so-called "excess of high- $Q^{2}$ events" in HERA experiments [20 high-energy scatterings of positrons and protons. Such an excess, if experimentally observed in a convincing way, might conceivably be attributed to the virtual effects of squarks in a $R$-parityviolating supersymmetric theory, according to

$e^{+}+d^{-, \frac{1}{3}} \rightarrow \operatorname{virtual} \tilde{t}^{\frac{2}{3}}\left(\operatorname{or} \tilde{c}^{\frac{2}{3}}\right) \rightarrow e^{+}+d^{-\frac{1}{3}}$,

for example. But let us close this parenthesis, since the reality of the effect has not been confirmed.

Altogether a large number of experimental studies have been devoted, since 1978, to searches for these new particles, most notably at $e^{+} e^{-}$ and $p \bar{p}$ colliders. The quest still remains unsuccessful, which implies lower limits on superpartner masses, generally of the order of $70-$ $80 \mathrm{GeV} / c^{2}$ for the various sleptons and winos, and more than a hundred $\mathrm{GeV} / c^{2}$ 's, for the strongly-interacting squarks and gluinos [2 [1] When can we finally expect to detect these longawaited superpartners? We may still have to wait for the Large Hadronic Collider at CERN, or maybe even a future very-high-energy linear $e^{+} e^{-}$collider. But the mass scale at which these new particles should be found is normally expected to be of the order of the electroweak scale, or up to a few $\mathrm{TeV} / c^{2}$ at most, if we do not want a new large mass scale associated with the superpartners to create a hierarchy problem in the electroweak theory.

\subsection{Grand unification}

Grand-unification theories [1] $[15]$ are invariant under larger gauge groups such as the $S U(5)$ of Georgi and Glashow (considered as the prototype of a grand-unification gauge group), or bigger groups like $O(10)$ or $E(6)$. They contain as subgroups both the color $S U(3)$ gauge group of the strong interactions and the $S U(2) \times U(1)$ gauge symmetry group of electroweak interactions. This leads to the possibility of relating the three corresponding gauge couplings, $g_{s}, g$ and $g^{\prime}$.

Once the various symmetry generators are normalized according to the same convention, which leads to consider the suitably-normalized gauge couplings

$g_{3} \equiv g_{s}, \quad g_{2} \equiv g$ and $\quad g_{1}=\sqrt{\frac{5}{3}} g^{\prime}$,

the grandunification symmetry imposes, above an appropriate energy scale called the grand unification scale $\left(m_{X}\right)$, the equality of the three couplings $g_{i=1,2,3}$. This would imply, in particular, $\tan \theta=g^{\prime} / g=\sqrt{3 / 5}$, and therefore ${ }^{10}$

$$
\sin ^{2} \theta=\frac{3}{8} \text {. }
$$

But the equality of the three gauge couplings $(15.5)$ - and the resulting value $\sin ^{2} \theta=3 / 8$ - should only be valid at the very high grandunification scale, at which electroweak and strong interactions are expected to have the same intensity. This is of course not the case at present energies, but one has to take into account the evolution of the three gauge couplings between their "low-energy" values, presently known at the energy scale of about $100 \mathrm{GeV}$, and the very highenergy scale $m_{X}$ at which the grand unification would start to become manifest.

All three gauge couplings are in fact slowlyvarying functions of the energy, whose values may be extrapolated from the present "low" energies (i.e. $\approx m_{Z}$ ) to very high energies, if the particle spectrum is known. For any given particle content assumed to be known one can derive, from the values of $\alpha\left(m_{Z}\right)$ and $\alpha_{s}\left(m_{Z}\right)$, the expected values of the electroweak mixing angle $\theta$ and of the grand-unification scale $m_{X}$, an essential ingredient in the determination of the expected lifetime of the proton.

\footnotetext{
${ }^{10}$ This value of $\sin ^{2} \theta$ may be obtained directly by expressing that $g T_{3}$ and $e Q$ appear as two $S U(5)$ generators with the same normalisation, which requires that $g^{2} \operatorname{Tr} T_{3}{ }^{2}=e^{2} \operatorname{Tr} Q^{2}$. Computing the trace for the fifteen chiral quark and lepton fields in one family, we have $\operatorname{Tr} T_{3}^{2}=8 \frac{1}{4}=2, \operatorname{Tr} Q^{2}=\frac{16}{3}$, and therefore $\sin ^{2} \theta=e^{2} / g^{2}=3 / 8$, at the grand unification scale.
} 
The minimal version of the $S U(5)$ model came close to a successful determination of $\sin ^{2} \theta$ $(\simeq .214 \pm .004)$, but there is now a significant discrepancy with the measured value, $\sin ^{2} \theta \simeq .231$. In other terms the three gauge couplings, determined from their now well-known "low-energy" values (with $\sin ^{2} \theta\left(m_{Z}\right) \simeq .231, \alpha\left(m_{Z}\right) \simeq$ $\left.1 / 129, \alpha_{s}\left(m_{Z}\right) \simeq .12\right)$, fail to meet at a common unification scale: there is no high-energy unification of the couplings. Furthermore the partial lifetime for the decay of the proton into $\pi^{0} e^{+}$was initially expected around $410^{29}$ years, in the simplest $S U(5)$ model (for which $m_{X} \simeq$ ( 1 to 4 ) $10^{14} \mathrm{GeV} / c^{2}$ ), with an estimated upper bound of about $310^{31}$ years. But experiments found no candidate for this decay mode, which implies a partial lifetime larger than $10^{33}$ years [22,]. This would require the grandunification mass $m_{X}$ to be larger than $\simeq 10^{15}$ $\mathrm{GeV} / c^{2}$, a result also in disagreement with the minimal version of the $S U(5)$ model.

The situation changes drastically, however, if the effects of the new particles of the supersymmetric standard model are taken into account. They lead to modified expectations for the values of the electroweak mixing angle $\theta$ and of the grand-unification mass $m_{X}$ [23]]. The unification scale is increased to about $\approx 10^{16} \mathrm{GeV} / c^{2}$, mostly as an effect of the gluinos and the $S U(2)$ gauginos, which slow down or even reverse the evolution of the non-abelian gauge couplings $g_{3}$ and $g$ of $S U(3)$ and $S U(2)$. At the same time the two Higgs doublets, with their associated higgsinos, modify the expected value of $\sin ^{2} \theta$, making it in good agreement with the one experimentally measured.

In the framework of grand-unification we now have an indirect indication that we may be on the right track. Superpartners and Higgs bosons would have a crucial influence on the evolution of the three $S U(3) \times S U(2) \times U(1)$ gauge couplings, and are essential to obtain their convergence at high energy - around $10^{16} \mathrm{GeV}$ or so - with the measured values of $\alpha_{s}\left(m_{Z}\right) \simeq 0.12$ and $\sin ^{2} \theta \simeq 0.231$. This convergence, obtained for the particle content of the Supersymmetric Standard Model with the new particles at their expected mass scale of $\approx 100 \mathrm{GeV}$ to $1 \mathrm{TeV}$ or so, may be taken, optimistically, as an indication in favor of the existence of these superpartners, and as a unification test of the Supersymmetric Standard Model.

\section{About new neutral currents, and (very) light gauge bosons}

\subsection{Searching for new neutral gauge bosons}

A large amount of very precise data has been accumulated over the years from parity-violation atomic physics experiments, polarized electronnucleon scattering experiments, neutrino-scattering and $e^{+} e^{-}$and $p \bar{p}$ scattering experiments, etc.. These data allow for very precise tests of the standard model, and a precise determination of $\sin ^{2} \theta$ which is essential for the discussion of grand-unified theories, as we just saw. They also lead to restrictions on the existence of possible new neutral gauge bosons $Z^{\prime}$ coupled to additional weak neutral currents. Fermilab.

One is generally used to think of new neutral gauge bosons as being very heavy, as they should in left-right symmetric models (with gauge group $\left.S U(2)_{L} \times S U(2)_{R} \times U(1)_{B-L}\right)$, or in grand-unified theories with larger gauge groups such as $O(10)$ or $E(6)$. All such models include at least one additional neutral gauge boson $Z^{\prime}$, with gauge couplings to ordinary fermions roughly of the order of the $S U(2) \times U(1)$ gauge couplings $g$ and $g^{\prime}$, as for the $Z$ couplings. There are, also, additional charged $W^{\prime \pm}$ bosons coupled to righthanded currents. ¿From the non-observation of such new $Z^{\prime}$ bosons in $p \bar{p}$ scattering experiments at Fermilab, one can deduce lower limits on their masses, which may reach about 600 $\mathrm{GeV} / c^{2}$, depending on the hypothesis made. Similar limits also exist for the additional $W^{\prime \pm}$ 's.

It is worth to keep in mind, however, the much less conventional possibility of new neutral gauge bosons that we call $U$ bosons, that could be much lighter, and possibly even extremely light, provided their gauge coupling $g$ " is, at the same time, relatively weak, or even extremely weak 24.7 . In the case of light $U$ bosons we can in general no longer evaluate exchange amplitudes 
in the local limit approximation as we often do for $Z$ bosons, and it is essential to take into account propagor effects.

To compare the magnitudes of $Z$-exchange and $U$-exchange amplitudes, let us recall that neutral current effects of the $Z$ boson are fixed by

$$
\frac{g^{2}+g^{\prime 2}}{q^{2}-m_{Z}^{2}} \simeq-\frac{8 G_{F}}{\sqrt{2}} \simeq-\frac{4}{v^{2}},
$$

which is proportional to $G_{F}$, in the local limit approximation for which $\left|q^{2}\right| \ll m_{Z}^{2} . \quad v=$ $\left(G_{F} \sqrt{2}\right)^{-\frac{1}{2}} \simeq 246 \mathrm{GeV}$ is often called the electroweak symmetry-breaking scale (c.f. footnote

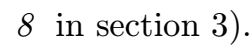

In a similar way, for a heavy $U$ boson with gauge coupling $g$ ", and a mass $m_{U}$ written proportionally to $g$ " $F, F$ denoting the extra- $U(1)$ symmetry-breaking scale (say $m_{U}=g " F / 2$, to keep things simple), the corresponding amplitude may be written similarly, again in the local limit approximation, proportionally to:

$$
\left.\frac{g^{\prime \prime 2}}{q^{2}-m_{U}^{2}} \simeq-\frac{4}{F^{2}} \quad \text { (at lower }\left|q^{2}\right| \ll m_{U}^{2}\right) \text {. }
$$

$U$-exchange amplitudes are then of the same order of magnitude as $Z$-exchange amplitudes, up to a scale factor

$$
\begin{aligned}
& r^{2}=\frac{v^{2}}{F^{2}} \\
= & \left(\frac{\text { electroweak scale }}{\text { extra } U(1) \text { symmetry breaking scale }}\right)^{2} .
\end{aligned}
$$

This ratio may be rather small, provided the corresponding new symmetry is broken at a scale $F$ sufficiently high compared to the electroweak scale $v \simeq 250 \mathrm{GeV}$, as it happens if an extra Higgs singlet acquires a large vacuum expectation value $\approx F \gg v$. (When the new coupling $g$ " is of the same order as $g$ or $g^{\prime}$, this simply means, not surprisingly, that $U$-exchange amplitudes are small compared to $Z$-exchange amplitudes, provided the $U$ boson is sufficiently heavy compared to the $Z$.)

The situation is, however, different in the case of relatively light or very light weakly-coupled
$U$ bosons, for which the local limit approximation is in general no longer valid [24]. We then have (in magnitude)

$$
\begin{aligned}
\frac{g^{\prime 2}}{q^{2}-m_{U}^{2}} & =\frac{g^{\prime 2}}{m_{U}^{2}} \frac{m_{U}^{2}}{q^{2}-m_{U}^{2}} \simeq \frac{g^{\prime 2}}{q^{2}} \\
& \ll \frac{4}{F^{2}} \text { for } m_{U}^{2} \ll\left|q^{2}\right| \cdot \quad
\end{aligned}
$$

As a result experiments performed at higher values of $\left|q^{2}\right|$ (compared to $m_{U}^{2}$ ) may well be insensitive to the existence of such light $U$ bosons, while experiments performed at lower $\left|q^{2}\right| \lesssim$ $m_{U}^{2}$, such as for example atomic physics experiments, would have a much better sensitivity. As an illustrative example for an extra $U(1)$ symmetry broken at or around the electroweak scale (i.e. $F \approx v$ ), atomic physics experiments performed with heavy atoms can be quite sensitive to the parity-violation effects induced by a $U$ boson, provided its mass is larger than about 1 $\mathrm{MeV} / \mathrm{c}^{2}$ [25] $]$. Such a $U$ boson, on the other hand, could have escaped detection in scattering experiments performed at higher values of $\left|q^{2}\right|$, owing to the $m_{U}^{2} /\left(q^{2}-m_{U}^{2}\right)$ reduction factor in the expression of $U$-exchange amplitudes.

The situation, however, again deserves further attention if the new gauge boson is really a very light $U$ boson, so that $m_{U}^{2}$ is practically always negligible, even in atomic physics parityviolation experiments. The amplitudes, proportional to $g^{\prime \prime} / q^{2}$, are, again, extremely small owing to the very small value of the gauge coupling $g "$, and even seem to vanish in the limit $g " \rightarrow 0$. U-boson effects would then seem to be totally negligible, in this limit. This, however, is not necessarily always the case. $U$-boson exchanges could still lead to a new long range force, that might be detected through apparent violations of the Equivalence Principle. Furthermore particle physics experiments themselves could be sensitive to such particles, even if the corresponding gauge coupling $g$ " becomes arbitrarily small, a somewhat surprising statement !

\subsection{A new long-range force?}

The possible extra $U(1)$ symmetries that could be gauged, in addition to the weak hypercharge $U(1)$, depend on the set of Higgs doublets responsible for the electroweak breaking. After 
mixing effects with the $Z$ boson are taken into account, the resulting $U$ current involves in general both vector and axial parts. The vector part is generally associated with the charge

$$
Q_{5}=x B+y_{i} L_{i}+z Q_{e l} .
$$

This vector part in the $U$ current would be responsible for a new force, acting on ordinary neutral matter in an additive way, proportionally to a linear combination of the numbers of protons and neutrons, $Z$ and $N$. If the $U$ boson is massless or almost massless with an extra $U(1)$ gauge coupling $g$ " extremely small, the new force would superpose its effects to those of gravitation, leading to apparent violations of the Equivalence Principle, since the numbers of neutrons and protons in an object are not exactly proportional to its mass $\left[24^{\prime}, \overline{2} \overline{6}^{\prime}\right]$. Newton's $1 / r^{2}$ law of gravitation could also seem to be violated, if the new force has a finite range.

If they have an appropriate magnitude, such violations of the Equivalence Principle could be detectable by the STEP experiment [20'] (Satellite Test of the Equivalence Principle). Violations of this principle could also be due to massless or quasimassless spin-0 particles, such as the "dilaton" of some superstring inspired models [2플. By monitoring the relative motion of two test masses of different compositions ${ }^{11}$ circling around the Earth, in a drag-free satellite, this experiment aims at testing the validity of this principle at a level of precision that could reach $\sim$ $10^{-17}-10^{-18}$, an improvement of five orders of magnitude compared to the present situation, in which this principle is known to be valid at a level of precision of about $10^{-12}$. Testing, to a very high degree of precision, the Equivalence Principle in space would bring new constraints on the possible existence of such forces, and might con-

\footnotetext{
${ }^{11}$ The test masses, however, cannot be taken as spherical, but only cylindrical. A potential difficulty is the existence of residual interactions between their higher multipole moments and the gravity gradients induced by disturbing masses within the satellite. This could simulate a "violation of the Equivalence Principle". To minimize these effects one can use test masses approaching ideal forms of "aspherical gravitational monopoles", which are (homogeneous) solid bodies for which all higher multipole moments vanish identically, despite the lack of spherical symmetry 29 i].
}

ceivably lead to a spectacular discovery, should a deviation from this Principle be found.

\subsection{A very light spin-1 $U$ boson does not decouple for vanishing gauge coupling!}

Let us now discuss whether it could make sense to search for a spin-1 $U$ boson with an extremely small gauge coupling $g$ ", in particle physics experiments. No one, however, would imagine being able to search directly for gravitons in a particle physics experiment, due to the extremely small value of the Newton constant $\left(\simeq 10^{-38}\right.$, in units of $\mathrm{GeV}^{-2}$ ). Then how could we search directly, in particle decay experiments, for $U$ bosons with even smaller values of the corresponding coupling, $g^{\prime \prime 2} \ll 10^{-38}$ ? Still this turns out to be possible! This rather astonishing result involves an "equivalence theorem" between the interactions of spin-1 particles and those of spin-0 particles, in the limit of very small gauge couplings [2]

One might think that, in the limit of vanishing extra- $U(1)$ gauge coupling constant $g$ ", the effects of the new gauge boson would be arbitrarily small, and may therefore be disregarded. But in general this is wrong, as soon as the $U$-current involves a (non-conserved) axial part! The amplitudes for emitting a very light ultrarelativistic $U$ boson, proportional to $g$ ", seem indeed to vanish with $g$ ". This is, however, misleading, since the polarization vector for a longitudinal $U$ boson, $\epsilon^{\mu} \simeq k^{\mu} / m_{U}$, becomes singular in this limit: $m_{U}=\frac{1}{2} g$ " $F$ also vanishes with $g$ ". Altogether the amplitudes for emitting, absorbing, or exchanging a longitudinal $U$ boson become independent of $g$ " when the $U$ boson is ultrarelativistic. Such a $U$ boson does then behave like a spin-0 particle ${ }^{12}$. This "equivalence

\footnotetext{
${ }^{12}$ The effects of the longitudinal $\left(q^{\mu} q^{\nu}\right)$ term in the $U$ boson propagator are essential in the limit considered, for which $m_{U}^{2} \ll\left|q^{2}\right|$. $U$-exchange amplitudes are then proportional to$$
\frac{g^{\prime \prime 2}}{q^{2}-m_{U}^{2}}\left(g^{\mu \nu}-\frac{q^{\mu} q^{\nu}}{m_{U}^{2}}\right) \rightarrow-\frac{4}{F^{2}} \quad \frac{1}{q^{2}} q^{\mu} q^{\nu} .
$$

One reconstructs the amplitudes for exchanging a massless spin-0 Goldstone particle, with derivative couplings proportional to $1 / F$. Applied to (non-conserved) axial currents $\bar{f} \gamma^{\mu} \gamma_{5} f$, the $q^{\mu} q^{\nu}$ terms regenerate the pseudoscalar Yukawa couplings of the equivalent spin-0 parti-
} 
theorem" expresses that in the low-mass or highenergy limit (i.e., for $m_{U} \ll E$ ), the third degree of freedom of a massive $U$-boson continues to behave like the massless spin-0 Goldstone boson which was "eaten away". For very small $g$ " the spin-1 $U$-boson simply behaves as this massless spin-0 Goldstone boson.

Incidentally the same phenomenon, in the case of local supersymmetry, called supergravity, expresses that a very light spin- $\frac{3}{2}$ gravitino (the superpartner of the spin-2 graviton), having interactions fixed by the gravitational "gauge" coupling constant $\kappa=\sqrt{8 \pi G_{N}} \simeq 4.110^{-19}$ $(\mathrm{GeV})^{-1}$, would behave very much like a massless spin- $\frac{1}{2}$ goldstino [1] 1 . Just like the mass of the $U$ boson was given in terms of the extra $U(1)$ gauge coupling $g$ " and symmetry breaking scale $F$ by the formula $m_{U}=\frac{1}{2} g$ " $F$, the mass of the spin- $\frac{3}{2}$ gravitino is fixed by its (known) "gauge" coupling constant $\kappa$ and the (unknown) supersymmetry-breaking scale parameter $d$, as follows:

$m_{3 / 2}=\frac{\kappa d}{\sqrt{6}} \simeq 1.68\left(\frac{\sqrt{d}}{100 \mathrm{GeV}}\right)^{2} 10^{-6} \mathrm{eV} / c^{2}$.

The interactions of a light gravitino are in fact determined by the ratio $\kappa / m_{3 / 2}$, or $G_{N} / m_{3 / 2}^{2}$, so that a sufficiently light gravitino interactions might be detectable in particle physics experiments, despite the extremely small value of the Newton constant $G_{N} \simeq 10^{-38}(\mathrm{GeV})^{-2}$, provided the supersymmetry-breaking scale ${ }^{13} \sqrt{d}$ is not too large. The gravitino would then be the lightest supersymmetric particle, with all other superpartners expected to ultimately produce a

$\overline{\text { cle, proportional to } 1 /} F$ times quark or lepton masses.

${ }^{13}$ An equivalent notation makes use of a parameter $\sqrt{F}=\sqrt{d} / 2^{1 / 4}$, defined so that $F^{2}=d^{2} / 2$, and therefore

$m_{3 / 2}=\frac{\kappa F}{\sqrt{3}} \simeq 2.37(\sqrt{F} / 100 \mathrm{GeV})^{2} 10^{-6} \mathrm{eV} / c^{2}$.

Furthermore, the supersymmetry-breaking scale $(\sqrt{d}$ or $\sqrt{F}$ ) associated with a (stable or quasistable) light gravitino should in principle be smaller than a few $10^{6} \mathrm{GeV}$ 's, for its mass to be sufficiently small $\left(m_{3 / 2} \lesssim 1 \mathrm{keV} / c^{2}\right)$, so that relic gravitinos do not contribute too much to the energy density of the Universe. gravitino among their decay products, if $R$-parity is conserved. (In particular the lightest neutralino could decay into photon + gravitino, so that the pair-production of "supersymmetric particles" could lead to final states including two photons with missing energy carried away by unobserved gravitinos.)

For sufficiently light gravitino one can also search for the direct production of a single gravitino associated with an unstable photino $\tilde{\gamma}$ (or more generally a neutralino), decaying into gravitino $+\gamma$, in $e^{+} e^{-}$annihilations. Or for the radiative pair-production of two gravitinos in $e^{+} e^{-}$ or $p \bar{p}$ annihilations at high energies [30 $\left.{ }^{\prime}\right]$, e.g.

$$
\begin{aligned}
& \quad e^{+} e^{-}(\text {or } p \bar{p}) \rightarrow \gamma(\text { or jet }) \\
& +\quad 2 \text { unobserved gravitinos }
\end{aligned}
$$

which have cross-sections

$\sigma \propto \frac{G_{N}^{2} \alpha\left(\text { or } \alpha_{s}\right) s^{3}}{m_{3 / 2}^{4}} \propto \frac{\alpha\left(\text { or } \alpha_{s}\right) s^{3}}{d^{4}}$.

Although the existence of so light gravitinos may appear as relatively unlikely, such experiments are sensitive to gravitinos of mass $m_{3 / 2} \lesssim 10^{-5}$ $\mathrm{eV} / c^{2}$, corresponding to supersymmetry-breaking scales smaller than a few hundreds of GeV's.

Just in the same way as the magnitude of $\sqrt{d}$ determines the effective strength of the gravitino interactions (and whether or not we may have a chance to produce it directly in particle physics experiments), the magnitude of the extra $U(1)$ symmetry-breaking scale parameter $F$ determines the effective strength of the $U$ boson interactions, for a $U$ coupled to a non-conserved current, as it is the case if this current includes an axial part. (A $U$ boson coupled to a conserved current does effectively decouple in the limit of vanishing gauge coupling.)

For such an extra $U(1)$ gauge symmetry broken at the electroweak scale ( $F \approx 250 \mathrm{GeV}$ ) by two Higgs doublets, the spin$1 U$-boson acquires essentially the same effective pseudoscalar couplings to quarks and leptons as a "standard" spin-0 axion. Its existence is then excluded by the results of $\psi(c \bar{c}) \rightarrow \gamma+$ "nothing" and $\Upsilon(b \bar{b}) \rightarrow \gamma+$ "nothing" decay experi- 
ments $\left[2 \overline{4}_{1}^{1}, \overline{2}-\overline{6}, \overline{3} \overline{1} 1\right]$. They imply that the extra$U(1)$ symmetry should be broken at a scale $F$ at least of the order of twice the electroweak scale - $\forall$.- -If,-on- the-other hand,-the-extr-a- $U$-(1) is broken "at a large scale" $F$ significantly higher than '- the electroweak scałe $\left(F^{-} \gg-250^{-} G e^{-} V^{-}\right)^{-=} u \operatorname{using}$ for example a very large Higgs-singlet vacuumexpectation-value, $U$-boson effects in particle physics would be practically "invisible". This mechanism relying on a large Higgs singlet v.e.v. , -and_applied to an extra global $U(1)_{\mathrm{P}_{\mathrm{Q}}}$-symmetry '- broken -at a-rory high-scato-alse allewect us to in

- dicate- that -axion interactiens in particte- physics could be made very small, with this axion mostly

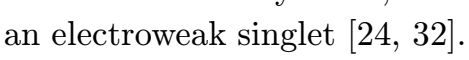

Such light $U$ bosons were initially discussed in the framework of supersymmetric theories with an extra $U(1)$ gauge group, but their possible - existence- shoutd be-considered-independently-of- -

- this originat motivation. To chose this special section dealing with new light bosons, let us also - mentien that the exchenges ef- a -new- spin-1- $L$

- boson; or of -a-spin-0-particte-such-as-the-axion, - could lead_to_new forces_acting_on particle spins '- including -a (CP-conserving) spin=spin interaction, and possibly a very small ( $C P$-violating) "mass-spin coupling" interaction.

\section{Conclusion}

Experiments on Parity Violation in electronhadron electroweak interactions brought spectacular contributions to our knowledge of the properties of the fundamental interactions, and con- tributed greatty to estabtish the vatidity - of -the - Standard Model- -But we- certainly-expect new - physics- beyond- the- Standard Model.- -Very prei eise tests -of-this model -give preciouts- infermations about the mechanism of electroweak symmetry breaking, and constraints on new physics beyond the standard model: existence of new gauge bosons, grand-unification, supersymmetry, ... This requires the study of many different

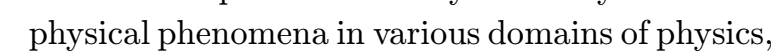
from very low to very high energies, and from microscopic physics to astrophysics and cosmology.

\section{References}

[1] S.L. Glashow, 'Nucl. Phys. B 22 $(1961) 579$; A. Salam and J.C. Ward, Phys. Lett. B $\mathbf{1 3}(1964)$

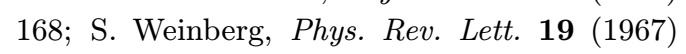
1264; A. Salam, Proc. 8th Nobel Symp., ed. N. Svartholm, (1968) 367.

[2] S. Weinberg, 'Rev. Mod. Phys. 52 (1980) $515 ;$ A. Salam, iRev. Mod. Phys. $\mathbf{5 2}(1980) 525 ;$ S.L. Glashow, 'Rev. Mod. Phys. 52 (1980) 539!'

[3] F. Englert and R. Brout, Phys. Rev. Lett. 13' (1964) 321' P.W. Higgs, 'Phys. Lett. B 12 (1964)', 132 'Phys. Rev. Lett. 13 (1964) 508.

[4] F.J. Hasert et al., 'Phys. Lett. B $\mathbf{4 6}(1973)$ 121; Phys. Lett. B 46 (1973) 138; A. Benvenuti et al., 'Phys. Rev. Lett. 32 $(1974) 800 !$.

[5] G. Arnison et al., 'Phys. Lett. B $\mathbf{1 2 6}$ (1983) 398; P. Bagnaia et al., Phys. Lett. B 129 (1983) 130.

[6] Ya. B. Zel'dovich, Sov. Phys. JETP $\mathbf{3 6}(1959)$ 964 [Sov. Phys. JETP 9 (1959) 682]; F. Curtis Michel, 'Phys. Rev. B 138 (1965) 408

[7] C.Y. Prescott et al., 'Phys. Lett. B $\mathbf{7 7}$ (1978)' 347 Phys. Lett. B $84(1979) 524$

[8] M.A. Bouchiat and C. Bouchiat, Phys. Lett. Bi $48(1974)-111_{i}^{\prime}$ and in ref. 9.

[9] "Parity Violation in Atoms and Polarized Electron Scattering" (Proc. of the Int. Conf. "ParityViolation in Electron-Hadron Electroweak Interactions", oct. 1998, Paris), B. Frois and M.A. Bouchiat eds., World Scientific, Singapore (1999); see in particular the contributions by G. Altarelli, M. J. Ramsey-Musolf, M.A. Bouchiat and C. Bouchiat, and R.D. McKeown.

[10] C.S. Wood et al., Science 275 (1997) 1759; V.A. Dzuba, V.V. Flambaum and O.P. Sushkov, preprint hep-ph/9709251.

[11] J. Erler and P. Langacker, 'Eur. Phys. J. $\left(\overline{1998)} 9 \overline{0}_{\mathrm{r}}^{1}\right.$

[12] The Super-Kamiokande collaboration, Phys.' Rev. Lett. 81 (1998) 1562.

[13] S. Weinberg, 'Phys. Rev. Lett. $\mathbf{4 0}(1978) 223$ F. Wilczek, Phys. Rev. Lett. 40 (1978) 279.'.

[14] A. Sakharov, Pis'ma Zh. Eksp. Teor. Fiz. 5 (1967) 32; 'S- Sov. Phys. JETP $\mathbf{5}(1967) 24$ '.

[15] H. Georgi and S.L. Glashow, 'Phys. Rev. Lett.' 32 (1974) 438; H. Georgi, H.R. Quinn and S. Weinberg, Phys. Rev. Lett. 33 (1974) 451; P.

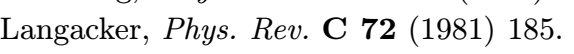


[16] Yu. A. Gol'fand and E.P. Likhtman, ZhETF Pis. Red.131971452; Sov. Phys. JETP $\mathbf{1 3}(1971)$ 323; D.V. Volkov and V.P. Akulov, 'Phys. Lett.' B 46 (1973) 109. J. Wess and B. Zumino, iNucl.i Phys. B 70 (1974) 39i; Supersymmetry and Su-

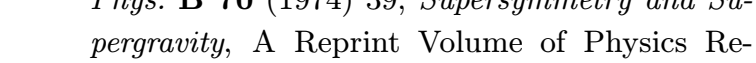
:----entiflc, 1980).

[17] P. Fayet, Lett. $\mathbf{B}-\overline{70}(1977)$ 461; in Histo

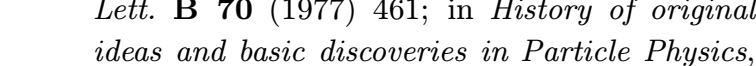
eds. H. Newman and T. Ypsilantis, Proc. Erice Conf., NATO Series B 352, 639 (Plenum, NY, 1996); G.R. Farrar and P. Fayet, Phys. Lett. Bi - - 76 (1978) 575

[18] See e.g. M. Green, J. Schwarz and E. Witten, Superstring theory, Cambridge University Press (1987).

[19] T.K. Hemmick et al. 'Phys. Rev. D_ 41 2074; P. Verkerk et al., 'Phys. Rev. Lett. 68' - _ - (1992) 1116!

[20] H1 Collaboration, 'Z. Physik C 4 (1997) 191; ZEUS Collaboration, 'Z. Physik $\mathbf{C} \mathbf{4}(1997) 20 \overline{7}_{1}$.

[21] Review of Particle Properties, 'Eur. Phys. J. Ci -

[22] R. Becker-Szendy et al., 'Phys. Rev. D 42 (1990), 2974; K.S. Hirata et al., Phys. Lett. B 220! (1989) 308; the Super-Kamiokande collaboration, 'Phys. Rev. Lett. $\mathbf{8 1}\left(\overline{1998)} 3 \overline{3} 1 \overline{9}_{1}^{\prime}\right.$.

[23] See e.g. U. Amaldi, W. de Boer and $H$. Fürstenau, iphys. Lett. B $\mathbf{2 6 0}(1991) 447 ;$ P. Langacker and M. Luo, ', Phys. Rev. D 44 (1991)', $---\overline{81} \overline{1}$

[24] P. Fayet, Phys. Lett. B 95 (1980)_285; 'Nucl.' - _ - Phys. B $\mathbf{1 8 7}(1981) 184$.

[25] P. Fayet, 'Phys. Lett. B 96 (1980) 83; C. Bouch; - - - - iat and C.A. Piketty, Phys. Lett. B 128 (1983)i

[26] P. Fayet, 'Nucl. Phys. B 347 (1990) 743; Class. Quantum Grav. 13(1996)A19.

[27] T. Damour and A.M. Polyakov, iNuch. Phys. - - $\mathbf{4 2 3}(1994) 532$.

[28] MiniSTEP, NASA-ESA report (1996); see also STEP, Satellite Test of the Equivalence Principle, Report on the Phase A Study, ESA SCI (96) 5 (1996).

[29] A. Connes, T. Damour and P. Fayet, iNucl. Phys.' - _ - 490 (1997) 391!
[30] P. Fayet, 'Phys. Lett. B 117 (1982) 460; 'Phys.। Lett. B $175(1986) 471_{1}$;. Brignole et al., $N u c l .1$ Phys. B 516_(1998) 13; iNucl. Phys. B 526. (1998) 136.

[31] C. Edwards et al., 'Phys. Rev. Lett. 48 (1982)' 903: Crystal Ball Collaboration, 'Phys. Lett. B' 251 (1990) 204 CLEO Collaboration, IPhys.' Rev. D $51(1995) 2053$

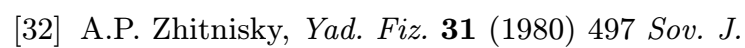
Nucl. Phys. 31 (1980) 260 M. Dine, W. Fischler and $\mathrm{M}$. Srednicki, 'Phys. Lett. B 104 (1981) 1991. 